\title{
Pierre Janin, Alain Marie (éds), « Violences
} ordinaires »

Politique africaine, 91, oct. 2003, 213 p.

\section{Sylvie Ayimpam}

\section{OpenEdition \\ Journals}

Édition électronique

URL : http://journals.openedition.org/apad/3663

DOI : 10.4000/apad.3663

ISSN : 1950-6929

Éditeur

LIT Verlag

Édition imprimée

Date de publication : 2 décembre 2003

Référence électronique

Sylvie Ayimpam, « Pierre Janin, Alain Marie (éds), «Violences ordinaires » », Bulletin de l'APAD [En ligne], 26 | 2003, mis en ligne le 16 juin 2008, consulté le 23 septembre 2020. URL : http:// journals.openedition.org/apad/3663; DOI : https://doi.org/10.4000/apad.3663

Ce document a été généré automatiquement le 23 septembre 2020.

Bulletin de I'APAD 


\section{Pierre Janin, Alain Marie (éds), «Violences ordinaires »}

Politique africaine, 91, oct. 2003, 213 p.

\section{Sylvie Ayimpam}

1 Ce dossier de la revue Politique africaine consacré à la violence sous ses aspects ordinaires, quotidiens, a le mérite de rompre avec la manière dont la violence en Afrique est généralement abordée: sous l'angle des violences politiques, des répressions, des rébellions, des guerres civiles ou ethniques. Pierre Janin et Alain Marie qui voient la violence comme « une coercition morale ou physique 'légitimée' par le droit du plus fort ", (p.5) soulignent dans leur introduction au thème, que la violence dont se chargent les relations politiques au sens strict, n'est pas dissociable de celle qui imprègne l'ensemble des rapports sociaux. Il est ainsi nécessaire de porter l'attention sur des «microprocédures" de pouvoir, donc de violence, dont on peut soutenir qu'elles sont enracinées dans la longue durée historique et donc dans la culture. L'espace domestique, lieu de la vie quotidienne dans toute sa banalité, et les structures familiales se présentent dès lors comme champ où se déploient ces microdispositifs ou microprocédures qui participent à la construction de ces systèmes de prédispositions structurées-structurantes déterminant des styles de conduites transposables dans des champs sociaux tels que celui de la politique stricto sensu et de ses violences spécifiques (Janin et Marie).

2 Les violences ordinaires, si souvent cachées dans un espace social comme la famille, doivent parfois être cherchées, débusquées, dans des pratiques devenues banales, tant l'on y est habitué. Là, elles s'expriment de manière tantôt larvées, tantôt explicites. La famille, généralement vue comme un espace de solidarité, n'assure pas toujours ses fonctions protectrices, ni dans les rapports de ses membres avec l'extérieur, ni dans leurs interrelations. Pierre Janin se pose d'ailleurs la question de savoir, comment la communauté familiale - microcosme de production du « social » de la société globale peut générer autant de violence normée ou anomique tout en la banalisant, à moins que la violence ne soit une forme d'intensification concurrentielle des relations sociales? (p. 35). 
3 En dehors de l'espace domestique, il y a aussi des violences ordinaires, que l'on peut trouver dans des rapports de voisinage, professionnels, d'affinité, etc. Des affrontements ordinaires, les violences ordinaires des gens ordinaires, (entre agriculteurs et pasteurs, élèves et enseignants, parents d'élèves et enseignants, etc.) peuvent parfois être des visages pris en temps de paix, comme c'est le cas pour le Tchad, de relations d'hostilité ayant opposé des sociétés étatiques et des sociétés segmentaires tout au long d'une histoire où la guerre et l'esclavage ont laissé des traces profondes dans les mentalités et les comportements, quel que soit le niveau d'éducation ou le statut social des protagonistes (p.66). Une telle violence, dans le cas du Tchad, a son enracinement dans la longue durée historique. Elle peut produire des antagonismes et se banaliser au point que certaines de ses manifestations, soient perçues par beaucoup, comme relevant du fonctionnement ordinaire de la société (p. 52).

4 Mais l'enracinement dans la longue durée historique ne concerne pas que les affrontements devenus ordinaires du genre de ceux que Claude Arditi décrit pour le Tchad. Elle atteint aussi la logique communautaire des rapports sociaux, encore prégnante et présente dans beaucoup de sociétés urbaines ou rurales d'Afrique. Le principe totalitaire qui gouverne les sociétés communautaires, se décline en violences permanentes faites à l'individu (Marie, p.14). Le microcosme villageois donne à percevoir dans la longue durée une violence ordinairement silencieuse des relations sociales les plus ordinaires : entre les hommes et les femmes et les aînés et les cadets. En milieu urbain, on observe qu'elle tend à devenir réciproque et à s'extérioriser, lorsque les individus réagissent par la violence à la violence supposée de leur communauté (p. 14-15). Cette forme d'autoritarisme sociétal qui régule les relations dans les sociétés de type communautaire et l'exercice d'une violence coercitive - qui ne requiert pas l'adhésion explicite des dominés, mais au besoin les contraint par l'usage de la force brute ou des pouvoirs occultes - peuvent permettre de comprendre que les sociétés africaines soient des sociétés violentes, animées en permanence par des rapports de force sous-jacents aux hiérarchies instituées, traduit fidèlement dans l'idiome de la sorcellerie (p. 8).

5 L'ordre communautaire, qu'il soit de type traditionnel ou moderne, fabrique de la violence réactive en chaque individu, le prédisposant aussi bien à subir la violence du groupe qu'à y concourir (Marie, p. 31). À son tour, chacun est abuseur et abusé ; il n'y a pas de responsabilité établie une fois pour toutes dans la répartition et la diffusion de la violence, puisqu'il n'y a pas de position dominante unilatérale et irréversible (Janin, p. 49). En milieu urbain, les dominés et les faibles, les cadets et les femmes, essaient de réagir contre la violence, puisque "là où il $\mathrm{y}$ a du pouvoir, il y a de la résistance ». L'usage que les femmes font de la nouvelle loi sur les violences domestiques à Westbury à Johannesburg en est une bonne illustration (Hornberger).

6 Dépassant le cadre marital traditionnel et incluant les relations occasionnelles et toutes les formes de liens familiaux, cette loi élargit le champ de la violence domestique et permet aux victimes de déposer des plaintes pour des faits pouvant aller de l'entrée non autorisée, passant par le harcèlement sexuel, le viol, les blessures corporelles graves, à la violence psychologique et l'intimidation (p. 84-85). En portant plainte et en retirant la plainte avant que le dossier ne soit transmis au tribunal, il s'agit vraisemblablement pour les femmes de faire peur aux maris afin de les dissuader d'exercer la violence sur elles. La réinterprétation qu'elles font de cette loi, leur permet de s'en servir comme d'une véritable arme de dissuasion contre les violences, en 
utilisant les policiers comme des travailleurs sociaux pour essayer de diminuer les tensions domestiques. C'est une interprétation «subtile des instruments fournis par l'Etat comme moyen susceptibles de régler des conflits sociaux localement prioritaires » (p. 89-90).

7 Les relations dominants-dominés, en dehors de l'espace domestique, peuvent prendre plusieurs visages, comme les rapports propriétaires/locataires dans les quartiers urbains. Marie Ange Goux offre une analyse de la réaction face à la coercition, à propos de la guerre des loyers dans les bidonvilles de Nairobi. Des propriétaires, souvent très puissants et liés aux milices armés, étouffent les protestations des locataires, contraints d'accepter toute augmentation de loyer, dégradation des locaux et densification du bâti, sans aucune possibilité de recours (p. 71-72). Ce qui aurait dû être une lutte de classes (riches propriétaires contre pauvres locataires) se transforme en une guerre des générations, entre jeunes locataires et vieux propriétaires, avec en arrière fonds des tensions ethniques et des rivalités politiques. La récupération des conflits par les politiciens se présentant en défenseurs des locataires, débouche sur des affrontements sanglants des bandes armées. Les locataires se constituent alors en organisations, s'introduisent dans l'arène politique pour la défense de leurs intérêts et mettent de la sorte fin à la domination des propriétaires sur les locataires en zones d'habitat pauvre (p.82). L'analyse de Marie-Ange Goux illustre comment la violence d'essence communautaire peut être cet invariant anthropologique universel utilisé fort bien par la volonté de puissance du politique « moderne » à ses fins propres (Marie, p. 31).

De la lecture de ce dossier, se dégage une impression d'inachevé. Les contributions sont très riches, mais en nombre insuffisant pour traiter les contours multiples et tentaculaires d'une question comme celle des violences ordinaires. D'ailleurs, les éditeurs eux mêmes appellent à poursuivre cette œuvre d'exploration dans une optique pluridisciplinaire. En dépit du fait que les contributions ont porté sur des pays appartenant à diverses régions de l'Afrique, Côte d'ivoire, Burkina Faso, Bénin, Tchad, Afrique du Sud, Kenya, il serait intéressant que d'autres études de cas puissent être faites. Elles permettraient sans doute d'explorer et/ou d'approfondir d'autres facettes des violences ordinaires qu'il n'était peut-être pas possible de discuter en un seul dossier, telle l'épineuse question explorée dans ce dossier, celle du rapport des violences ordinaires aux violences politiques.

\section{AUTEUR}

\section{SYLVIE AYIMPAM}

Doctorante à l'Institut d'Études du Développement de l'Université Catholique de Louvain, Louvain-la-Neuve, Belgique 